\title{
Quantum tunneling in magnetic systems of various sizes (invited)
}

B. Barbara, L. C. Sampaio, J. E. Wegrowe, B. A. Ratnam, and A. MarchandC. Paulsen, M. A. Novak, and J. L. TholenceM. UeharaD. Fruchart

Citation: Journal of Applied Physics 73, 6703 (1993); doi: 10.1063/1.352508

View online: http://dx.doi.org/10.1063/1.352508

View Table of Contents: http://aip.scitation.org/toc/jap/73/10

Published by the American Institute of Physics

Save your money for your research.

It's now FREE to publish with us -

no page, color or publication charges apply.

Publish your research in the

Joumal of Applied Physics

to claim your place in applied

physics history. 


\title{
Quantum tunneling in magnetic systems of various sizes (invited)
}

\author{
B. Barbara, L. C. Sampaio, J. E. Wegrowe, B. A. Ratnam, and A. Marchand \\ Laboratoire Louis Néel, BP166, 38042, Grenoble, France \\ C. Paulsen, M. A. Novak, and J. L. Tholence \\ Centre de Recherches sur Les Très Basses Températures, BP166, 38042, Grenoble, France \\ M. Uehara \\ National Research Institute for Metals, 1-2-1, Sengen, Tsukuba, Ibaraki 305, Japan \\ D. Fruchart \\ Laboratoire de Cristallographie, BP166, 38042, Grenoble, France
}

\begin{abstract}
Magnetic relaxation experiments constitute a unique method of determining the nature of fluctuations in dissipative magnetic systems. At high temperatures these fluctuations are thermal and strongly temperature dependent. At low temperatures, where quantum fluctuations dominate, magnetic relaxation becomes independent of temperature. Such behavior has been observed in many systems. In this review we emphasize the study of low temperature relaxation in ferromagnetic nanoparticles, layers, and multilayers (including "domain wall junctions"), and large single crystals. The results of magnetic relaxation experiments are shown to agree with theoretical predictions of quantum tunneling of the magnetization. When dissipation becomes important, in large and complex systems, a time dependent WKB exponent needs to be introduced.
\end{abstract}

\section{INTRODUCTION}

More than sixty years after the creation of quantum mechanics, the analysis of its foundations still continues to be a very active field of research. ${ }^{1}$ Recent advances in mesoscopic physics, made possible by new technologies of miniaturization and by the development of highly sensitive SQUID magnetometry, have contributed to a renewed interest in this field of research. Some years ago, mesoscopic physics was limited to transport measurements. Now mesoscopic physics deals more and more with magnetism. After the discovery of persistent currents, ${ }^{2,3}$ which can be viewed as coherent diamagnetism, we are now on the road to the discovery of similar quantum-coherent phenomena in ferromagnetism, ferrimagnetism, antiferromagnetism.

Few attempts have been made to investigate quantum tunneling of the magnetization (QTM) in the spirit of mesoscopic physics (see D. Awschalom et al. ${ }^{4}$ and this symposium). This approach is certainly the most interesting, though rather difficult. The effect of environmental spins on the large (tunneling) moment has been considered recently by Leggett ${ }^{1}$ and Stamp ${ }^{5}$ in the framework of the difference associated with the parity of these moments. ${ }^{6,7} \mathrm{It}$ seems that the large number of environmental spins might destroy the quantum coherence.

A different approach to QTM is the measurement of the temperature dependence of the magnetic relaxation right after an abrupt change in the applied magnetic field. This technique known as "magnetic after-effect," is morc than 50 years old ${ }^{8,9}$ and is now commonly used to determine particle size distributions, energy barrier distributions, activation volumes, and more generally, to analyze the process of magnetization reversal (see e.g., Ref. 10). To the best of our knowledge, Bean and Livingstone ${ }^{11}$ and Weil ${ }^{12}$ were the first to propose QTM as a possible alternative explanation of an upturn of the particle size distribution at low energies (leading to a bimodal distribution). The present understanding of QTM, however, suggests that it would have been difficult to demonstrate quantum effects in nickel due to its weak anisotropy. A few years later, magnetic after-effect experiments have been performed on several rare-earth-based (highly anisotropic) systems. ${ }^{13}$ These experiments showed two important features (i) fast magnetic relaxation at low temperature, attributed to very narrow domain walls (thickness of a few interatomic distances) and (ii) energy barriers proportional to the reciprocal applied field, $E \alpha 1 / H$, attributed to domain wall motion via 2-dimensional nucleations on the wall surface. This latter mechanism was motivated by analogy with ferroelectric domain wall motion (Ref. 13 and references therein). In the same year, 1973, Egami ${ }^{14}$ formalized this interpretation in two models of 2-d nucleation: thermal or quantum nucleation of narrow domain walls with intrinsic pinning. Since 1975 , several papers devoted to the static and dynamic properties of $\mathrm{SmCo}_{3,5} \mathrm{Cu}_{1,5}$ single crystal have appeared. In particular a crossover temperature from thermal activation to quantum tunneling of about $10 \mathrm{~K}$ (Ref. 15) have been interpreted by a phenomenological model in which domain walls are pinned by point defects. ${ }^{16}$ Similar results showing a crossover temperature of $5 \mathrm{~K}$ on $\mathrm{Dy}_{3} \mathrm{Al}_{2}$ single crystal (dominated by intrinsic pinning) was interpreted ${ }^{17}$ in terms of Egami's theory (see Fig. 1). These early results on $\mathrm{Dy}_{3} \mathrm{Al}_{2}$ are no less suggestive of QTM than several of the more recent ones. However, as recently mentioned by Stamp, ${ }^{18}$ the modern story of QTM really starts in 1986 with the detailed analysis of the $\mathrm{SmCo}_{3.5} \mathrm{Cu}_{1.5}$ (Ref. 15) experiments. The effects of dissipation and possible sample heating in these bulk systems have been considered later. ${ }^{44}$

With regard to QTM theory, we should mention that theory is now more advanced than the experiments. Excepting the two papers of Egami and a paper of Chudnovsky (1979), ${ }^{19}$ theory really started in $1986-88$ with the papers of Chudnovsky and Gunther ${ }^{20}$ and Enz and Schilling. ${ }^{21}$ Decisive breakthroughs were made by Stamp with 


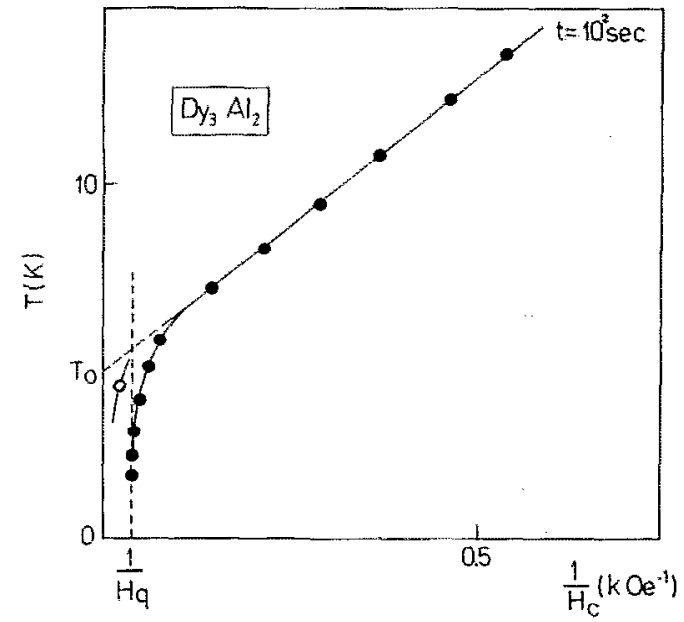

FIG. 1. Thermal variation of the coercive field of $\mathrm{Dy}_{3} \mathrm{Al}_{2}$ at the time scale of $10^{2} \mathrm{~s}$. The point $(0)$ has been taken at $4.2 \mathrm{~K}$ and $10^{-6} \mathrm{~s}$. The low temperature plateau with a crossover temperature at $5 \mathrm{~K}$ is time dependent (from Ref, 17).

the evaluation of the QTM probability for a domain wall with dissipation. ${ }^{22}$ In general, theoretical predictions converge on the idea of extremely weak dissipation effects in magnetic systems of nanometric dimensions (see, e.g. Ref. $23)$. Finally, one must say that the general ideas of macroscopic quantum tunneling (MQT), developed by Leggett in the early 80 's, ${ }^{24}$ led the way for most, if not all of the theoretical studies, and certainly stimulated experimental searches for MQT.

In this paper we show that nonthermal relaxation effects, similar to those obtained in $\mathrm{Dy}_{3} \mathrm{Al}_{2}$ and $\mathrm{SmCo}_{3.5} \mathrm{Cu}_{1.5}$ single crystais ${ }^{15,17}$ exist in a large number of diverse systems. In Sec. II we give a comprehensive study of two systems of nanoparticles ( $\mathrm{TbCeFe}_{2}, 150 \mathrm{~A}$ and $\mathrm{FeC}, 20 \mathrm{~A}$ ) in which variations of the energy barrier with field, magnetization level, and temperature are determined down to $50 \mathrm{mK}$. Section III discusses some of the amorphous layer and multilayer results, and in particular, presents first results on domain wall junctions. In this progression from the nanoscopic to the macroscopic scale, we finally describe in Sec. IV, the dynamical magnetic and thermal behavior of some large ferromagnetic single crystals. These systems are extremely interesting to study the effects of dissipation in complex systems. They might show real MQT events at very short time scale.

\section{FERROMAGNETIC NANOPARTICLES}

\section{A. Experimental procedure}

General aspects of relaxation measurements in magnetic systems with thermal and quantum fluctuations are considered in Ref. 25. When the experimental system is sufficiently disordered, energy barriers are uniformly distributed (as in, e.g., amorphous alloys). We then measure the magnetic viscosity $S=d M / d \ln t$. In general, for nonuniform distributions, it is important to perform relaxation experiments at constant magnetization $M$ so that $M$ does not interfere with the evaluation of the energy barrier. ${ }^{15,25,29}$ We usually use $M=0$ and plot $d M / d t$ vs time. This defines the "mean" relaxation time $\tau$,

$$
1 / \tau=\left(1 / 2 M_{S}\right)(d M / d t)_{M=0} \text {. }
$$

This relaxation time $\tau$ coincides with the "median" relaxation time of the distribution, "most probable" relaxation time for a symmetrical distribution.

\section{B. Quantum tunneling of domain walls in $\mathrm{Tb}_{0.5} \mathrm{Ce}_{0.5} \mathrm{Fe}_{2}$ particles}

$\mathrm{Tb}_{0.5} \mathrm{Ce}_{0.5} \mathrm{Fe}_{2}$ particles were obtained by a reduction in $\mathrm{H}_{2}$ gas. They have a relatively large size distribution around a mean value of $150 \mathrm{~A}$ and a cubic symmetry. Crystal field effects acting on the total angular momentum give a large anisotropy of the free energy $\left(5 \times 10^{7} \mathrm{erg} /\right.$ $\left.\mathrm{cm}^{3}\right)$. The $\mathrm{Fe}-\mathrm{Fe}$ exchange energy is also important $\left(10^{9}\right.$ $\mathrm{erg} / \mathrm{cm}^{3}$ ). At zero field and in thermodynamical equilibrium, most of the particles will be single domain $\left(R_{c} \sim \gamma /\right.$ $M s^{2} \sim 10^{3}$ A). Near $H_{c}$, however, metastable configurations will introduce, on average, one domain wall (of thickness $\delta \sim 50 \mathrm{~A}$ ) per particle (details will be published elsewhere).$^{26}$ In our experiment the magnetization of the sample was first saturated in a field of $8 \mathrm{~T}$. The field was then decreased to zero, reversed, and stabilized at a given value of $H$ close to the coercive field $H_{c}$. The magnetic relaxation was then measured as a function of the applied field and temperature. The low temperature measurements were made using a high field/low temperature SQUID magnetometer and a moveable miniature dilution refrigerator. The recording of the decay of the magnetization was continuously measured for 1 to $2 \mathrm{~h}$ and the median relaxation time defined above was determined. Experimentally $\tau$ is directly related to the slope $(d M / d t)^{-1}$ of the $M(t)$ curve at $M=0$.

\section{Field dependence of the median energy barrier}

More than 70 different values of $\tau(H, T)$ were measured. These allow us to determine the field and temperature dependencies of the corresponding energy barrier, the median energy barrier (MEB). We have found that the field and temperature variations of $\tau(H, T)$ are well described by

$$
\tau(H, T)=\tau_{1} \exp \left[A\left(1 / H-1 / H_{0}\right) / k T^{*}\right],
$$

where $H_{0}$ is the maximum coercive field, $A$ is a constant, and $T^{*}$ is the effective temperature defined in Ref. 15 $\left(T^{*}=T\right.$ at high temperature and $T^{*}=T_{c}$ at low temperature). This expression is illustrated in Fig. 2. The straight lines represent the values of $1 / \tau$ calculated from the sum of the thermal activation (TA) and quantum tunneling (QT) rates [the $T^{*}(T)$ curve, which is extracted from this plot, is very close to the one given by the "quantum harmonic oscillator" formula]. ${ }^{15,27}$ The best fitting parameters are $1 / H_{0}=0.15 \pm 0.02$ and $\log 1 / \tau_{1}=8.5 \pm 0.5$ (coordinates of the focal point) as well as the crossover temperature $T_{c}=600 \mathrm{mK}$ were obtained by a rather sensitive scaling plot of $\log (d M / d t)$ vs $\left(1 / H-1 / H_{0}\right) / k T$ (this plot was very similar to the one given here in Fig. 5 for the magnetic viscosity). The measured data points for $T<400 \mathrm{mK}$ are clustered on a line very close to the calculated QTM limit (labeled MQT in Fig. 2). Note that these plots and their 


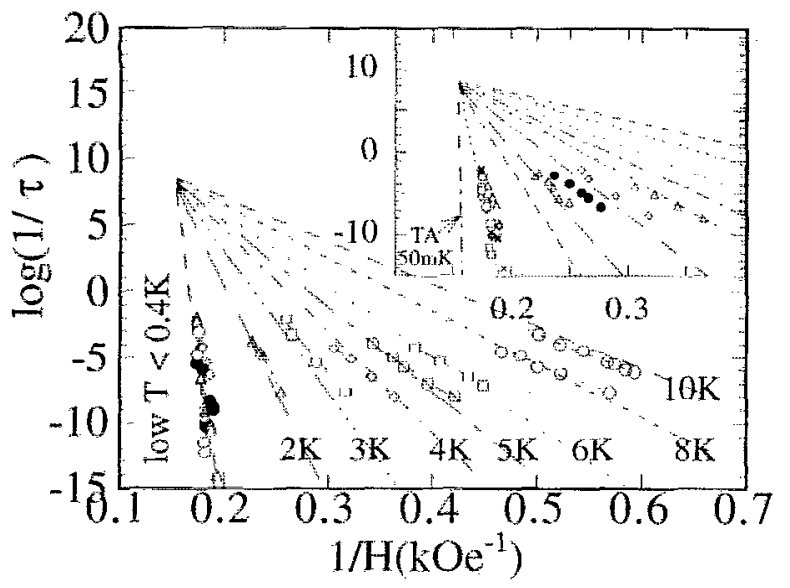

FIG. 2. $\log (1 / \tau)$ vs $1 / H$ in $\mathrm{Tb}_{0,5} \mathrm{Ce}_{3} \mathrm{Fe}_{2}$. The temperature range is $0.05 \%(K)-10$ and the low temperature data are at $T=400,200,100$, 66 , and $50 \mathrm{mK}$.

extrapolations are very similar to those which establish our present belief of MQT in Josephson junctions.

\section{Temperature dependence of the effective temperature $T^{*}(T)$}

The effective temperature $T^{*}$ is determined from the slopes of the lines representing each isotherm in Fig. 2, that is $1 / T^{*}=d \log (1 / \tau) / d(1 / H)$. The variations of $1 / T^{*}$, versus the reciprocal temperature $1 / T$, are represented in Fig. 3. At high temperatures a TA regime is observed where $T^{*}=T$. At temperatures below $600 \mathrm{mK}, T^{*}=T_{c}$ is practically independent of temperature. This is exactly what is expected in the general theory of MQT (Leggett ${ }^{1,24}$ as well as in theories of QTM in ferromagnetic systems. ${ }^{20-24,28}$ This is also consistent with our earlier QTM experiments on bulk ferromagnets (Ref. 15 and references therein).

\section{Temperature dependence of the tunneling volume}

This volume, analogous to the well known TA volume in thermal activation processes, can be evaluated at any

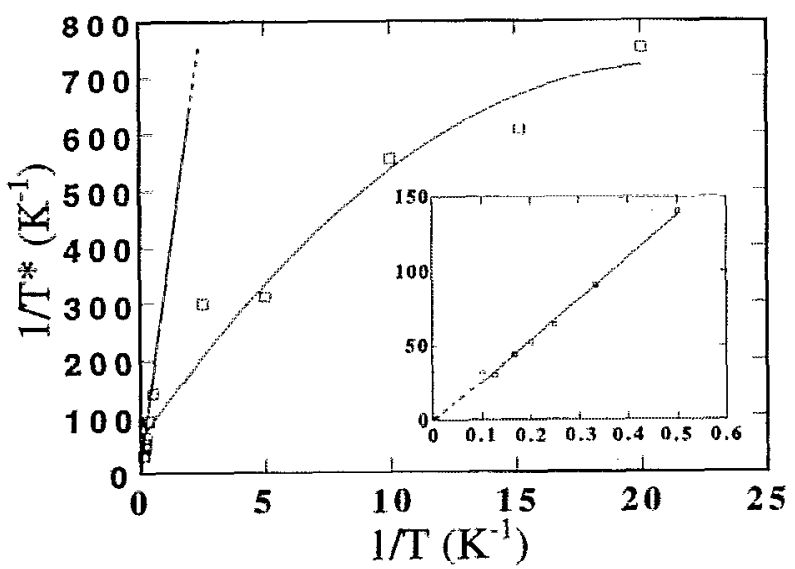

FIG. 3. $1 / T^{*}$ vs $1 / T$. The crossover temperature is $T_{\mathrm{c}}=0.6 \mathrm{~K}$. Inset: high temperature TA regime. Note that the TA regime extrapolates to $1 / T=0$.

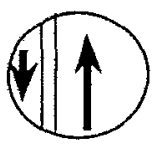

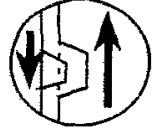

b

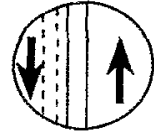

c
FIG. 4. Schematic representation of the supposed motion of a domain wall crossing a particle. A 2-d nucleation takes place on the domain wall (b) and the nucleated domain wall deformation moves from the center of the particle to its boundaries (soliton) (b) and (c).

temperature by equating the energy barrier $E=k T^{*} \ln (t)$ $\left.t_{0}\right)$ to the Zeeman energy at the maximum coercive field. ${ }^{15}$ This volume, $V-E / M S H-30 k_{B} T^{*} / M_{S} H$ is proportional to the temperature above $1 \mathrm{~K}$ (at $T=10 \mathrm{~K}, V=5 \times 10^{5}$ $\mathrm{A}^{3}$ ) and is constant below $1 \mathrm{~K}, V=2.10^{4} \mathrm{~A}^{3}$ ). The existence of such small volumes clearly shows that the dynamics of magnetization reversal is not uniform at the scale of each particle. The magnetization reversal of each particle proceeds by a succession of domain wall quantum jumps, each jump being initiated by a 2 -d quantum nucleation on its surface, followed by classical soliton motion (scheme Fig. 4). ${ }^{29,30}$

\section{Barkhausen noise}

The sum of all such local magnetization jumps in volumes $V$, leads, above the crossover temperature, to the well known nonequilibrium Barkhausen noise ( $\mathrm{BN}$ ). Below the crossover temperature, nonequilibrium $\mathrm{BN}$ dominates because local reversals of the magnetization are induced by QTM rather by TA. Note that "equilibrium BQN" is certainly also present due to the many QT events with correlation lengths smaller than the 2-d critical nucleation length: if the tunneling from say, the $A$ well to the $B$ well concerns a number of moments smaller than the critical size required for 2 -d nucleation, the elastic domain wall tension should drive the inverse tunneling from $B$ to $A$. Domain wall tension is equivalent here to negative dissipation and should increase the effects of coherence.

\section{Dissipation}

The QTM/TA crossover time $t_{c o}$ defined for bulk systems in Sec. IV is dramatically increased in nanoparticles. This is because the quasiparticles emitted at each QTM event should immediately be absorbed in the surrounding bath due to their extremely large surface/volume ratio. The effect of dissipation of different origins here is only related to the coupling of the order parameter to the degrees of freedom of the bath. Our experiments on small particles do not appear to show adverse effects of dissipation. Only a weak temperature dependence $T^{*}(T)$ in $\mathrm{Tb}_{0,5} \mathrm{Ce}_{0.5} \mathrm{Fe}_{2}$ which might well be due to the fact that the experiment was performed close enough to the coercive field, which may well have increased the effects of dissipation. ${ }^{31,32}$ The classical soliton-like motion which follows each QTM event is certainly weakly dissipative. The. "soliton" will come to rest immediately (Fig. 4), unlike in bulk samples where the classical motion, which takes the form of a catastrophic avalanche comes to rest after a discrete magnetization (and heat) pulse. 


\section{QTM in FeC particles}

This system is made up of a frozen ferrofluid. The particle sizes are slightly distributed about a very small diameter, $20 \mathrm{~A}$. We have used the same procedure as in the previous section and have obtained very similar results down to $100 \mathrm{mK} \cdot{ }^{33}$ The MEB also follows a $1 / H$ law and the effective temperature $T^{*}$ shows a well-defined plateau below $1 \mathrm{~K}$ down to $100 \mathrm{mK}$. The reason for the nonhomogeneous magnetization reversal, characterized by our $1 / H$ law, is the same as in $\mathrm{TbCeFe}_{2}$ particles. This confirms the large (surface) anisotropy of this system. Dipolar interactions certainly slow down the relaxation but it does not have an inhibitory effect, since local dipolar fields are smaller than anisotropy fields. However, the relatively narrow particle size distribution requires using the dynamics at constant $M,{ }^{15,25}$ rather than the magnetic viscosity. Interestingly, we find a tunneling volume of the order of $(20$ A), ${ }^{3}$ i.e., of order of the particle's size.

\section{MULTILAYERS AND DOMAIN WALL JUNCTIONS}

These systems have only one nanoscopic dimension, along the direction perpendicular to their planes. Dissipation effects should a priori, be larger than in nanoparticles but smaller than in bulk materials. However, several amorphous alloys seem to show weaker dissipation than in nanoparticles. We shall give a few reasons for this apparent contradiction.

\section{A. $\mathrm{Fe} / \mathrm{Ag}$ and $\mathrm{FeTb} / \mathrm{Cu}$ amorphous multilayers}

Both of these systems show a well-defined lowtemperature plateau in the "instantaneous magnetic viscosity," i.e., the tangent to the $M(\ln t)$ curve at time $t_{0}$ at which the experiment started. In the $\mathrm{Fe}(1000 \mathrm{~A}) / \mathrm{Ag}$ multilayer we have observed a plateau of the magnetic viscosity down to $50 \mathrm{mK} .{ }^{34}$ Other experiments performed on amorphous layers and multilayers including $\mathrm{FeTb} /$ $\mathrm{Cu}^{33,35-39}$ are limited to above $1.5 \mathrm{~K}$. In Ref. 25 we give several expressions which allow us to obtain the field dependence of the energy barrier $g\left(H / H_{0}\right)$ and of the crossover temperature $T_{c}(H)$. For $a-\mathrm{FeTb}(300 \mathrm{~A}) / \mathrm{Cu}$, we find $g\left(H / H_{0}\right)$ proportional to $1 / I I$ as in $a-\mathrm{TbAg}^{40}$ and multilayers of FeSm. ${ }^{36} \mathrm{~A}$ scaling plot of the normalized magnetic viscosity of $\mathrm{FeTb}(300 \mathrm{~A}) / \mathrm{Cu}$ is shown in Fig. 5. The fact that the $1 / H$ barrier does not vanish in finite fields suggests that elastic energy of interfaces is neglible, as expected for 2-d systems. $T_{c}$ of these 2-d alloys should be larger than those of 3-d systems. This is because magnetization reversal in these systems is associated with the nucleation and propagation of topological defects such as vortices, antivortices, and magnetic strings with very small inertial masses. ${ }^{41}$ However, the mean tunneling volume, $V-10^{3}$ atoms is independent of dimensionality.

\section{B. Realization of domain wall junctions}

A planar domain wall trapped by a planar defect thinner than the domain wall thickness is analogous to a Josephson junction (JJ). ${ }^{27}$ As a matter of fact, the $\mathrm{JJ}$ and DWJ equations for $\sin ^{2}$ (or cubic) potentials are identical.

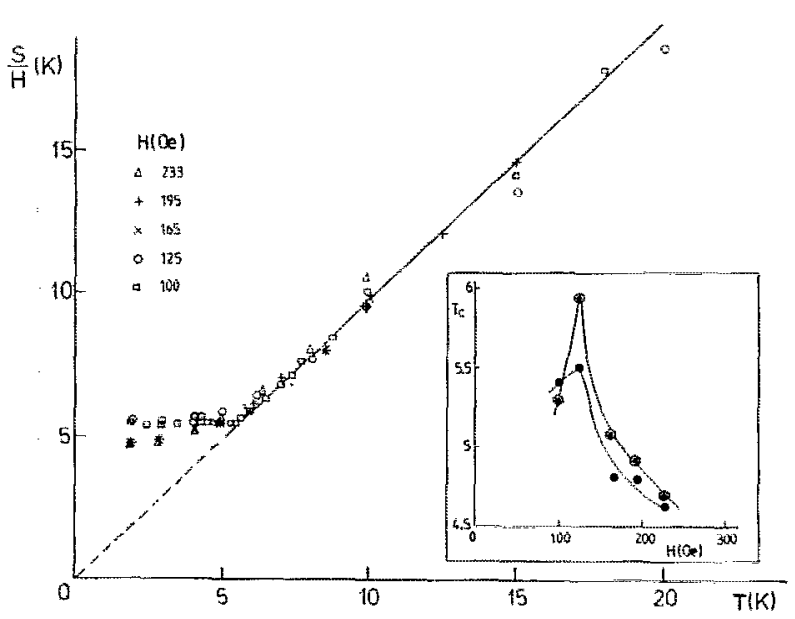

FIG. 5. Scaling of the magnetic viscosity normalized to temperature, in $a-\mathrm{Fe} \mathrm{Tb} / \mathrm{Cu}$, deduced from the $1 / H$ field dependence of the energy barrier. Data from Ref. 37. Inset: $T_{r}(H)$ obtained from nonscaled $(*)$ and scaled (๑) results.

For example DWJ recently modeled by Chudnovsky et al $^{42}$ give results analogous to those for $\mathrm{JJ}$. We have realized two types of junctions. In the first junction we have introduced an artificial energy barrier by increasing the anisotropy energy in a layer in sandwich between two ferromagnetic layers ( $b$-DWJ). In the second one, we have introduced an artificial energy well by decreasing the exchange energy in a layer in sandwich between two ferromagnetic layers ( $w$-DWJ). DWJ hysteresis loops exhibit unique features. A typical hysteresis loop in our $(\mathrm{Co} /$ $\mathrm{CoCu} / \mathrm{Co}) w$-DWJ junctions is shown in Fig. 6. The first smooth magnetization jump comes from the creation of a Bloch wall centered (pinned) on the planar artificial defect. The second one, stepper, results from the extraction of this wall out of this pinning plane. Magnetic relaxation experiments on this $\mathrm{Co} / \mathrm{CoCu} / \mathrm{Co}$ systen and on a $b$-DWJ made of thin layers of $\mathrm{GdFe}_{2}$ and $\mathrm{SmFe}_{2}$ are shown in Fig. 7. Details will be published in a forthcoming paper. ${ }^{43}$ Here we give the variations measured at a given well-defined time scale of (i) the magnetization in a constant small field $(15 \mathrm{Oe}$ ) (down to $50 \mathrm{mK}$ ) in the $b$-DWJ and (ii) the temperature dependence of the coercive field ( $M$ $=\mathrm{constant}=0$ ) in the $w$-DWJ. The two types of junctions

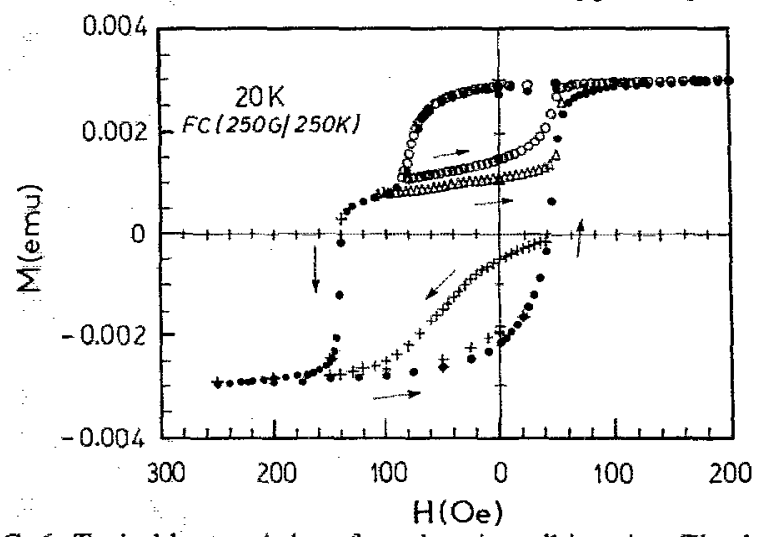

FIG. 6. Typical hysteresis loop for a domain wall junction. The domain wall nucleates along the first transition near -100 Oe and stays pinned on the planar defect between this field and the propagation field for which the macroscopic Bloch wall is extracted from the defect (here $155 \mathrm{Oe}$ ). This last jump can occur through QTM (Fig. 7). 
behave similarly with same hysteresis loops and relatively high crossover temperature (about $5 \mathrm{~K}$ ). The main difference between simple films and our junctions is essential: when our junctions were reduced to submicronic scales, QTM events of a single portion of domain wall were observed.

\section{BULK FERROMAGNETIC SINGLE CRYSTALS}

The effective temperature $T^{*}$ of a 3-mm single crystal of $\mathrm{SmCO}_{3.5} \mathrm{Cu}_{1,5}$ has a low tempcrature plateau. ${ }^{15}$ However, when the temperature is low enough, catastrophic heat pulses $(\Delta T=H d M / C)$ associated with giant magnetization jumps destroy subsequent QTM events that follow the first one. ${ }^{44}$ This leads to a staircase hysteresis loop where a time-dependent WKB exponent is expected. ${ }^{30}$ Results obtained on other bulk samples are also discussed.

\section{A. QTM and dissipation in $\mathrm{SmCo}_{3.5} \mathrm{Cu}_{1.5}$}

Nonthermal relaxation of the magnetization above 4 $\mathrm{K}$, shows a plateau below $10 \mathrm{~K}$. The curve $T^{*}(T)$ has been fitted to an expression where we assume additivity of the thermal and nonthermal relaxation rates ${ }^{15}$ and later ${ }^{27}$ fitted to the "harmonic oscillator formula," $T^{*}=T_{c} \operatorname{coth}\left(T_{c}\right.$ $T) .{ }^{15}$ Both fits give similar $T^{*}(T)$ dependencies. Interestingly, $\mathrm{JJ}$ data fits to the harmonic oscillator formula are excellent (Ref. 27 and references therein).

\section{Staircase hysteres/s loops and heat pulses in $\mathrm{SmCO}_{3.5} \mathrm{Cu}_{1.5}-$ Time-dependent WKB exponent and crossover temperature}

When the temperature of the bath is decreased to $2 \mathrm{~K}$, large discontinuities appear in the hysteresis loops of $\mathrm{SmCo}_{3.5} \mathrm{Cu}_{1.5}$. At a still lower temperature, the discontinuities become very similar and more numerous. At $1.8 \mathrm{~K}$ the hysteresis loop consists of a coherent set of magnetization jumps. The reversal of the (macroscopic) remanent magnetization of the single-crystal takes place through a staircase-like behavior. ${ }^{44}$ Each magnetization jump is initiated by a time-dependent mechanism, as is evidenced by stair shifts toward larger fields with faster sweep fields. Each jump is a step of about $1 \mu \mathrm{m}$ of the domain walls in the $(3 \mathrm{~mm})$ sample in a time $\tau_{1} \sim 1 \mathrm{~ms}$ that slows down in $\tau_{2} \sim 5 \mathrm{~ms}$. In a first approximation, $d M / d t \sim\left(t / \tau_{1}\right) \exp ($ $\left.-t / \tau_{2}\right)$. The simplest explanation of this phenomenon is obviously related to sample heating resulting from the transfer of the domain wall instantaneous power $H d M / d t$, to phonons, plasmons, spin-waves, etc. This phenomenon is observed at a low temperature where the specific heat $C$ is small, and the adiabatic increase of sample temperature $\Delta T=H \Delta M / C$ large. Classical domain walls motions, which immediately follow first quantum nucleations at the beginning of a given magnetization jump, lead to emissions of quasiparticles (phonons, spin-waves, etc.). After some delay, the crossover time $t_{\mathrm{co}}$ avalanche of quasiparticles spread throughout the sample giving temperature rises: TA events dominate QTM. If experiments are performed on time scales larger than $t_{\text {co }}$, QTM events observed below 10 $\mathrm{K}$ disappear below $2 \mathrm{~K}$, leading to reentrant QTM. However, QTM should be observable at smaller time scales. A more detailed analysis shows that there exist two time scales: $t_{\mathrm{co}}$ and $t_{s}$. For $t t_{c o}$, where zero-point vibrations of the magnetic degrees of freedom are decoupled from phonons and other excitations we have QT motion of domain walls, for $t_{\mathrm{co}}<t_{s}$ the domain wall motion is TA, with sample heating ${ }^{30}$ and for $t \gg t_{s}$ the classical motion slows and this is the end of the avalanche. ${ }^{26}$ These results are based on time-dependent dissipation. Starting from the Caldeira-Leggett expression ${ }^{32}$ our analysis finds, for this system, that the tunneling probability decreases with time as

$$
p_{Q t}=\left(\tau_{0} / \tau_{Q}\right) \exp -C\left(t / \tau_{1}\right)^{4},
$$

where $1 / \tau_{Q}$ is the tunneling probability in the absence of dissipation and $C$, a constant containing a damping coeffi cient and the barrier width. Our crossover time is defined by $p_{Q t}=p_{T}$ the TA probability. We find $t_{\mathrm{co}}=10^{-9} \mathrm{~s}$ at 1.8 $\mathrm{K}$ (an evaluation of $t_{\mathrm{co}}$ at $4 \mathrm{~K}$ requires extremely sensitive calorimetric measuring experiments, these experiments are currently underway). Our results are consistent with the picture in which the entire domain wall surface of the sample $(3 \mathrm{~mm})$ tunnels over the distance of its thickness $(20$ A) in $10^{-9} \mathrm{~s}$. This is subsequently followed by a succession of $10^{10} \mathrm{TA}$ events of similar size. Our understanding of such a MQT event needs to consider the domain wall elastic energy that preserves the identity of the domain wall structure by acting as a source of "negative dissipation."
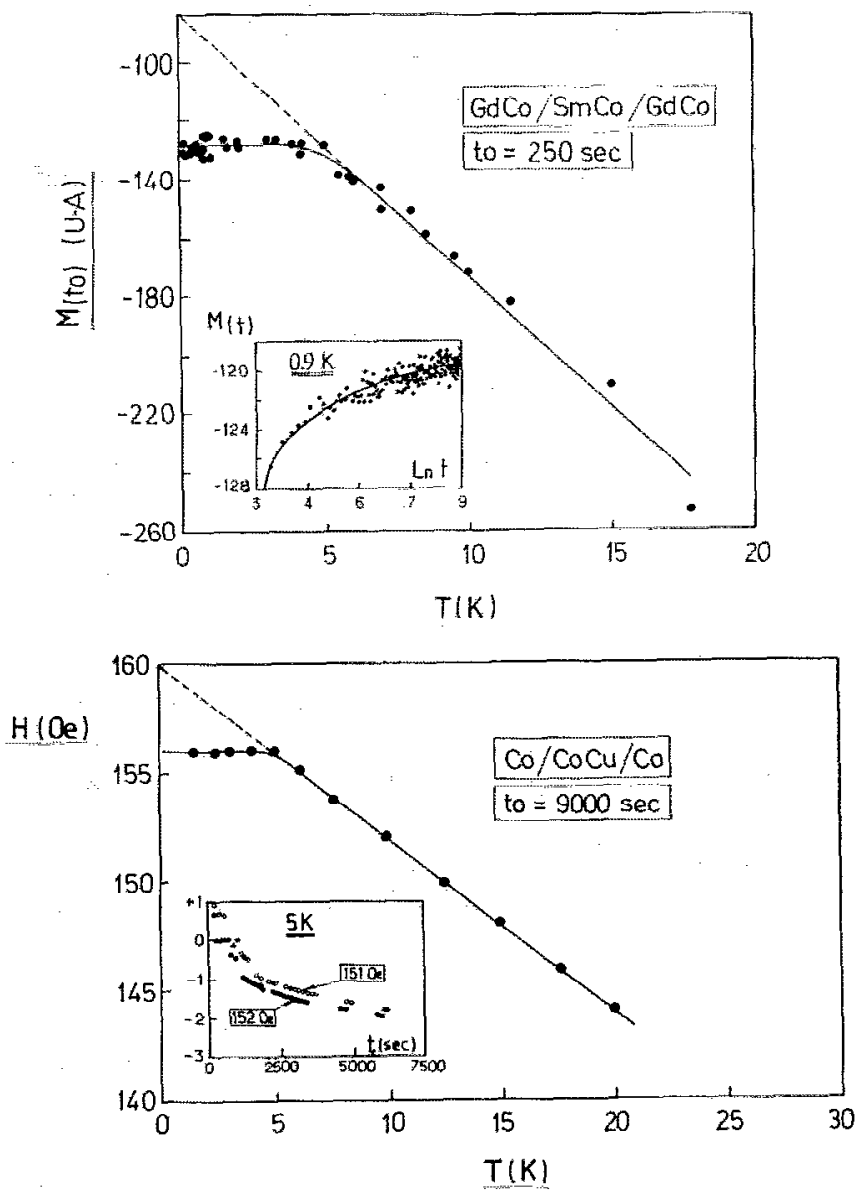

FIG. 7. First observation of a thermal to nonthermal crossover (near 5 $\mathrm{K}$ ) on the dynamics of a $b$-DWJ (a), and of a $w$-DWJ (b). Inset: time dependence. 


\section{B. Precursor oscillations in $\mathrm{Sm}(\mathrm{CoCuFeTi})_{6.8}$ and coupling to the bath}

Besides the smallness of low temperature specific heat, sample heating can also result from a poor exchange with the thermal bath. If the thermal contact between sample and bath is strongly reduced, precursor oscillations of both $d M / d H$ and $d T / d H$ appear. ${ }^{30}$ These oscillations are closely related to each other even when they exhibit chaotic behavior. Their origin is unclear though they evidence the connection between large magnetization jumps and quasiparticle emission that leads to sample heating. The fact that precursor oscillations are present only when the coupling of the sample with the thermal bath is reduced proves the importance of energy transfers from the sample to the bath through the sample surface.

\section{CONCLUSION}

Nonthermal relaxation of the magnetization, observed and interpreted in terms of QTM a decade ago (Ref. 15 and references therein), seems to now have been confirmed in several systems down to rather low temperatures (50 $\mathrm{mK}$ ). Are we sure that this is effectively QTM? In principle, it is possible that some distribution of energy barriers (e.g., a power law distribution as in self-organized criticality ${ }^{45}$ ) or sample heating could play a role. It is, therefore, extremely important to carefully analyze these effects. A detailed analysis of the general aspects of relaxation measurements in magnetic systems with thermal and quantum fluctuations ${ }^{25,29,46}$ shows that it is often important to determine the complete relaxation function $\tau(H, t, M)$. This is what we have done. The heating problem is more serious, especially if it occurs at space and time scales much smaller than experimental ones. In dissipative large systems we have observed heat pulses in the ms range. However, sample heating should come only after the time during which the "particle" stays out of equilibrium on the "escape excited level." This time is shorter if this excited level is broadened by couplings with other degrees of freedom such as phonons. Reference 30 shows that the heat pulse is much slower than the magnetization jump, in agreement with the adiabatic effect discussed by Leggett. ${ }^{47}$ This leads to a time-dependent WKB exponent $t_{0}$ and consequently to "time-limited QTM" in bulk (complex) systems at low temperature. Depending on the complexity of the system, this crossover time can vary over several decades. In small particles or thin layers, QTM events are sufficiently disconnected to each other so that this effect of time-dependent WKB exponent is negligible. However, these effects could give some size limitations in possible future applications of QTM (e.g., quantum computer, ${ }^{18}$ DWJ, etc.).

\section{ACKNOWLEDGMENTS}

B. B. is grateful to A. J. Leggett for an early correspondence on QTM. He also thanks the Research Laboratory of the Merlin et Gerin. Company for its support (contract No. 509855). L. C. S. and M. A. N. would like to acknowledge the leave of absence from CBPF-CNPq and UFRJ, respectively, and the support by $\mathrm{CNPq}$ fellowships (Brazil).
${ }^{1}$ A. J. Leggett, Proc. Int. Symp. on Foundations of Quant. Mech., Tokyo, 74 (1983). For the effect of environmental spins (private communication).

${ }^{2}$ M. Buttinker, Y. Imry, and R. Landauer, Phys. Lett. 96A, 365 (1983). ${ }^{3}$ P. Levy, G. Dolan, J. Dunsmuir, and H. Bouchiat, Phys. Rev. Lett. 64, 2074 (1990).

${ }^{4}$ D. D. Awschalom, M. A. McCord, and G. Grinstein, Phys. Rev. Lett. 65, 783 (1990); D. D. Awschalom, D. P. di Vincenzo, and J. F. Smith, Science 258, 414 (1992).

${ }^{5}$ P. C. M. Stamp, preprint (Aug. 1992).

${ }^{6}$ D. Loss, D. P. di Vincenzo, and G. Grinstein, Phys. Rev. Lett. 69, 3232 (1992).

${ }^{7}$ E. M. Chudnovsky, preprint (Aug. 1992).

${ }^{8}$ L. Néel, J. Phys. Rad. 12, 339 (1951).

${ }^{9}$ J. C. Barbier, Ann. Phys. Paris 9, 84 (1954).

${ }^{10} \mathrm{~B}$. Barbara and M. Uehara, IEEE Trans. Magn. Mag. 12, 6, 997 (1976).

"C. P. Bean and J. D. Livingstone, J. Appl, Phys. 30, 1205 (1959).

${ }^{12}$ L. Weil, J. Phys. Chem. 51, 715 (1954).

${ }^{13}$ B. Barbara, G. Fillion, D. Gignoux, and R. Lemaire, Solid State Commun. 10, 1149 (1972).

${ }^{14}$ T. Egami, Phys. Status Solidi A 20, 157 (1973); ibid. 19, 747 (1973).

${ }^{15}$ M. Uehara and B. Barbara, J. Phys. 47, 235 (1986).

${ }^{16} \mathrm{~B}$. Barbara and $M$. Uehara, Proc. Rare Earth Conf. Durham, Inst. Phys. 37, 203, (1978).

${ }^{17}$ B. Barbara, Proc. 2nd Int. Symp. on Coercivity, Cal. Univ., San Diego, 137 (1978).

${ }^{18}$ P. C. M. Stamp, Nature 359, 365 (1992).

${ }^{19}$ E. Chudnovsky, JETP 50, 1035 (1979).

${ }^{20}$ E. M. Chudnovsky and L. Gunther, Phys. Rev. Lett. 60, 661 (1988).

${ }^{21}$ M. Enz and R. Schilling, J. Phys. C 19, L-711 (1986).

${ }^{22}$ P. C. E. Stamp, Phys. Rev. Lett. 66, 2802 (1991).

${ }^{23}$ A. Garg and G. H. Kim, Phys. Rev. Lett. 63, 2512 (1989).

${ }^{24}$ A. J. Leggett, Rev. Mod. Phys. 59, 1 (1987); Supplement to Progress in Theoretical Physic 69, 80 (1980).

${ }^{25} \mathrm{~B}$. Barbara (unpublished).

${ }^{26}$ B. Barbara (unpublished).

${ }^{27}$ B. Barbara, M. Uehara, and P. C. E. Stamp, J. Phys. 49, C8-529 (1988).

${ }^{28}$ P. C. E. Stamp, E. M. Chudnorsky, and B. Barbara, Int. J. Mod. Phys. B6, 1355 (1992).

${ }^{29}$ C. Paulsen, L. C. Sampaio, B. Barbara, D. Fruchart, A. Marchand, J. L. Tholence, and M. Uehara, Phys. Tett. A 161, 319 (1991); EuroPhys. Lett. 1, 643 (1992). B. Barbara, Proc. Int. Workshop, Studies of magnetic properties of fine particles and their relevance to materials science, edited by Dormann and Fiorani (Elsevicr, Amsterdam, 1991).

${ }^{30}$ B. Barbara, L. C. Sampaio, A. Marchand, M. Uehara, C. Paulsen, J. L. Tholence, and D. Fruchart, Int. Symp. on Foundations of Quant. Mech. Tokyo (August 1992).

${ }^{31}$ E. Chudnovsky (these proceedings).

${ }^{32}$ A. O. Caldeira and A. J. Leggett, Phys. Rey. Lett. 46, 211 (1981); Ann. Phys. (N.Y,) 149, 374 (1983).

${ }^{33}$ L. Balcells, J. L. Tholence, S. Linderoth, B. Barbara, and J. Tejada, Z. Phys. B 89, 209 (1992).

${ }^{34}$ M. A. Novak, W. Wernsdorfer, B. Barbara, J. Tejada, and X. X. Zhang (unpublished).

${ }^{35} \mathrm{X} . X$. Zhang, $\mathrm{I}_{3}$. Balcells, J. M. Ruiz, O. Iglesias, J. Tejada, and B. Barbara, Phys. Lett. A163, 130 (1992).

${ }^{36}$ X. X. Zhang, L. Balcells, J. M. Ruix, J. L. Tholence, B. Barbara, and J. Tejada, J. Cond, Matter 4, L-163 (1992).

${ }^{37}$ X. X. Zhang, J. Tejada, and B. Barbara, submitted to the Int. Symp. on Mag. Thin Films and Multilayers (Lyon, France Sept. 1992).

${ }^{38} \mathrm{~J}$. Tejada (these proceedings).

${ }^{39} \mathrm{~J}$. J. Arnaudas, A. del Moral, and C. de la Fuente (unpublished).

${ }^{40}$ B. Boucher and B. Barbara, J. Phys. Colloq. 40, C5-240 (1979).

${ }^{41} B$. Dieny and B. Barbara, Phys. Rev. B 41, 11549 (1990).

${ }^{42}$ E. Chudnovsky, O. Iglesias, and P. C. E. Stamp (unpublished).

${ }^{43}$ J. E. Wegrowe, M. A. Novak, and B. Barbara (unpublished).

${ }^{44}$ M. Uehara, B. Barbara, B. Dieny, and P. Stamp, Phys. Lett. 114A, 23 (1986).

${ }_{45}^{45}$ Per Bak, C. Tang, and K. Wiesenfeld, Phys. Rev. Lett. 59, 381 (1987).

${ }^{4} \mathrm{~B}$. Barbara and L. Gunther, J. Magn. Magn. Mater. (to be published).

${ }^{47}$ A. J. Leggett, Int. Symp. on Foundations of Quant. Mech., Tokyo (Aug. 1992). 